\title{
Structural Characterisation and Mechanical Stability of Titanium Substituted Mesoporous Materials
}

\author{
${ }^{a}$ C. Galacho, ${ }^{b}$ M.M.L. Ribeiro Carrott, ${ }^{c} J$. Valerio, ${ }^{d}$ P.J.M. Carrott, \\ e J.M.V. Nabais
}
Centro de Química de Évora e Departamento de Química da Universidade de Évora, Rua Romão Ramalho $n^{\circ} 59$, 7000-671 Évora, Portugal
apcg@uevora.pt, ㄹmanrc@uevora.pt, cjoanaritavalerio@hotmail.com, dpeter@uevora.pt, jvn@uevora.pt,

Keywords:Ti-SBA-15, Mechanical stability, X-Ray diffraction (XRD), Nitrogen adsorption at 77K.

\begin{abstract}
Mesoporous silica and titanium SBA-15 samples with different Si/Ti molar ratio were prepared by direct one-step synthesis under strongly acidic hydrothermal conditions. The materials were characterised by X-ray diffraction, nitrogen adsorption at $77 \mathrm{~K}$. The $\mathrm{Si} / \mathrm{Ti}$ values of the final calcined materials were determined by ICP analysis. It was found that all samples presented 2-D hexagonal structures with high degree of ordering. However, the metal content incorporated in all titanium mesoporous materials was very low, i.e., the Si/Ti molar ratio values of the final calcined samples are significantly different from the initial $\mathrm{Si} / \mathrm{Ti}$ molar ratio in the synthesis gel. A study of mechanical stability in air was performed by exposing the calcined samples to a unidirectional external pressure in the range $74-814 \mathrm{MPa}$. X-ray diffraction and nitrogen adsorption at $77 \mathrm{~K}$, on selected compacted samples, were used in order to evaluate the changes in mesoporous structure. It was found that, for all freshly calcined pure silica and titanium containing samples tested, the high quality SBA-15 structure is still observed after the application $148-370 \mathrm{MPa}$ of a unidirectional external pressure and the collapse of the pore structure occurs at around $814 \mathrm{MPa}$.
\end{abstract}

\section{Introduction}

The discovery of TS-1 in the 1980s was a remarkable breakthrough in the synthesis of titanium containing materials. However, the substrates which can be oxidised in the presence of these microporous titanium silicates are limited to molecules with kinetic diameters less than $0.6 \mathrm{~nm}$. The availability of titanium mesoporous materials such as Ti-MCM-41 [1-3] and Ti-SBA-15 [4,5] broadens the potential applications of Ti-containing porous solids as catalysts in selective oxidation reactions, which are extremely important for the production of valuable intermediates in fine chemistry. These MCM-41 and SBA-15 type materials with an hexagonal array of unidirectional tubular pores, extremely high surface area and porosity, combine the advantages of presenting a narrow pore size distribution and having pore size adjustable from $\sim 2$ to $30 \mathrm{~nm}$. Nevertheless, heterogeneous catalytic oxidation processes and other fields of application can be substantially reduced due to lack of stability under process conditions. Thus the structural stability, namely, thermal, hydrothermal and mechanical, is a key factor in their practical applications.

Several studies have been published concerning the mechanical stability of the pure silica mesoporous materials, such as SBA-15 [6-8], and also materials organically functionalised by a direct method [9]. However, to the best of our knowledge there is only our previous work published concerning the mechanical stability of ordered mesoporous materials containing titanium, namely, Ti-MCM-41 [10]. Therefore, the work presented here focuses on the structural characterisation of Ti-SBA-15 materials, prepared by direct synthesis with different Si/Ti molar ratios, and their mechanical stability under application of unidirectional external pressure in the range 74-814MPa, which includes the industrial conditions for making pellets as the commonly applied pressure is in the range $10-400 \mathrm{MPa}[8]$. 